\title{
Safe implementation of robotic-assisted minimally invasive direct coronary artery bypass: application of learning curves and cumulative sum analysis
}

\author{
Jef Van den Eynde ${ }^{1,2,3} \wedge$, Hannah Vaesen Bentein ${ }^{1,2}$, Tom Decaluwé ${ }^{1,2}$, Herbert De Praetere ${ }^{1,2}$, \\ MaryAnn C. Wertan ${ }^{4}$, Francis P. Sutter ${ }^{4}$, Husam H. Balkhy ${ }^{5}$, Wouter Oosterlinck ${ }^{1,2}$ \\ ${ }^{1}$ Department of Cardiovascular Diseases, Research Unit of Cardiac Surgery, University Hospitals Leuven, Leuven, Belgium; ${ }^{2}$ Department of \\ Cardiovascular Sciences, KU Leuven, Leuven, Belgium; ${ }^{3}$ Helen B. Taussig Heart Center, The Johns Hopkins Hospital and School of Medicine, \\ Baltimore, MD, USA; ${ }^{4}$ Division of Cardiac Surgery, Lankenau Medical Center, Wynnewood, PA, USA; ${ }^{5}$ Division of Cardiac Surgery, Department of \\ Surgery, University of Chicago Medicine, Chicago, IL, USA \\ Contributions: (I) Conception and design: J Van den Eynde, H Vaesen Bentein, T Decaluwé, W Oosterlinck; (II) Administrative support: J Van den \\ Eynde, H Vaesen Bentein, T Decaluwé, W Oosterlinck; (III) Provision of study materials or patients: W Oosterlinck; (IV) Collection and assembly \\ of data: J Van den Eynde, H Vaesen Bentein, T Decaluwé, W Oosterlinck; (V) Data analysis and interpretation: J Van den Eynde; (VI) Manuscript \\ writing: All authors; (VII) Final approval of manuscript: All authors. \\ Correspondence to: Prof. Dr. Wouter Oosterlinck, MD, PhD. Department of Cardiovascular Diseases, University Hospitals Leuven, KU Leuven, \\ Herestraat 49, 3000 Leuven, Belgium. Email: wouter.oosterlinck@med.kuleuven.be.
}

Background: Learning curves are inevitably encountered when first implementing an innovative and complex surgical technique. Nevertheless, a cluster of failures or complications should be detected early, but not deter learning, to ensure safe implementation. Here, we aimed to examine the presence and impact of learning curves on outcome after robotic-assisted minimally invasive direct coronary artery bypass (RAMIDCAB).

Methods: A retrospective analysis of the first 300 RA-MIDCAB surgeries between July 2015 and December 2020 was performed. Learning curves were obtained via logarithmic regression for surgical time. Cumulative sum (CUSUM) analysis was performed for (I) major complications including MI, stroke, repeat revascularization, and mortality, and (II) other complications, including prolonged ventilation, pneumonia, pleura puncture, lung herniation, pericarditis, pleuritis, arrhythmia, wound complications, and delirium. Expected and unacceptable rates were set at $12 \%$ and $20 \%$, respectively, for major complications, and at $40 \%$ and $60 \%$ for other complications, based on historical data in conventional coronary artery bypass grafting (CABG).

Results: Demographic characteristics did not differ between terciles, except for more smokers in the first tercile, and less hypercholesterolemia and more complex procedures in the third tercile. The mean surgical time for all operations was $258 \pm 81$ minutes, ranging from 127 to 821 minutes. A learning curve was only observed in the first tercile. Subgroup analysis revealed that this learning curve was only observed for procedures consisting of single internal mammary artery (SIMA) with 1 or 2 distal anastomoses but not with bilateral internal mammary arteries (BIMA) or more than 2 distal anastomoses. CUSUM analysis showed that the cumulative rate of major and other complications never crossed the lines for unacceptable rates. Rather, the lower $95 \%$ confidence boundary was crossed after 50 cases, indicating improvement in safety.

Conclusions: These results suggest that integration of RA-MIDCAB in the surgical landscape can be safely achieved and complication rates can quickly be reduced below those expected in traditional CABG. Collective experience plays a key role in overcoming the learning curve when more complex procedures and cases are introduced.

^ ORCID: 0000-0002-5606-376X. 
Keywords: Cardiac surgery; coronary artery bypass grafting (CABG); learning curve; minimally invasive surgery; teaching

Submitted May 03, 2021. Accepted for publication Jun 11, 2021.

doi: $10.21037 /$ jtd-21-775

View this article at: https://dx.doi.org/10.21037/jtd-21-775

\section{Introduction}

The acquisition of new surgical skills and techniques is often associated with a learning curve. With increasing experience, surgical time usually shortens and less complications occur. Previous research has demonstrated the presence of learning curves in minimally invasive surgery of the mitral valve $(1,2)$. Furthermore, it has been shown that even in early stages of implementation of minimally invasive aortic valve replacement, comparable results could be achieved to conventional surgery (3). This illustrates the two-way importance of learning curve and cumulative sum (CUSUM) analyses for complications: these allow for safe monitoring during training of surgeons, and can help to temper reluctance of adopting new procedures.

Robotic-assisted minimally invasive direct coronary artery bypass (RA-MIDCAB) is an especially challenging procedure that is currently being introduced in several hospitals (4). Handling of the robot for internal mammary artery harvesting, operating on a beating hart, anastomosing "in the deep" through a small incision, all require advanced training. Due to the complex nature of the procedure and because safety is a major prerequisite for responsible introduction, we aimed to examine the presence of learning curves and their impact on surgical outcome.

We present the following article in accordance with the STROBE reporting checklist (available at https://dx.doi. org/10.21037/jtd-21-775) (5).

\section{Methods}

\section{Population and study design}

This study conforms to the ethical guidelines of the Declaration of Helsinki (as revised in 2013) as reflected in a priori approval by the local Ethical Committee of the University Hospitals of Leuven (No.: S64856). Informed consent was taken from all individual participants. A prospectively collected clinical database was retrospectively reviewed to identify the first 300 consecutive patients who underwent RA-MIDCAB at the University Hospitals of
Leuven between July 1, 2015 and December 31, 2019. Patients were followed up based on information available in their electronic medical records, as well as hospitalizations and outpatient surgical and cardiology consultations. Minimal period of follow-up for all patients was until discharge or death. The study period was divided into three terciles.

Demographics included age, gender, and body mass index (BMI). Medical comorbidities and risk factors such as obesity, smoking, diabetes mellitus, arterial hypertension, hypercholesterolemia, plasma creatinine levels, and EuroSCORE II, were also collected. Perioperative information contained the presence of previous percutaneous coronary intervention (PCI), admission for acute coronary syndrome, RA-MICAB as part of a hybrid procedure, type of construct, and surgical time. A hybrid procedure was defined as RA-MIDCAB either preceded or followed by PCI within 6 months of the operation. Type of construct was classified as procedures consisting of (I) single internal mammary artery (SIMA) +1 distal anastomosis, (II) SIMA +2 distal anastomoses, or (III) bilateral internal mammary arteries (BIMA) or more than 2 distal anastomoses.

To assess surgical safety, postoperative complications were collected and divided into two groups: major adverse cardiac and cerebrovascular events (MACCE) and other complications. MACCE was defined as the occurrence of myocardial infarction (MI), stroke, repeat revascularization, or mortality within 1 year after surgery. MI was defined in accordance with the Fourth Universal Definition of Myocardial Infarction [2018] (6). Repeat revascularization was defined as any surgical or interventional revascularization performed on previously treated vessels. Other complications consisted of a composite of representative complications after coronary surgery, including prolonged ventilation (defined as ventilation $>24 \mathrm{~h}$ ), pneumonia, pleura puncture, lung herniation, pericarditis, pleuritis, arrhythmia requiring pacemaker implantation, wound complications, or delirium. Pericarditis was defined as inflammation of the two layers 
of the thin, sac-like membrane that surrounds the heart, as confirmed based on electrocardiography, echocardiography, and/or treatment for pericarditis. Pleuritis was defined as inflammation of the tissue layers (pleura) lining the lungs and inner chest wall, as diagnosed by the characteristic chest pain which is aggravated by breathing, physical findings on examination of the chest and/or chest X-ray. Wound complications were defined as described in a previous report and adapted to thoracotomy (7). Briefly, wound complications ranged from mild and superficial wound problems or infections at the thoracotomy or port sites to severe and deep complications that need subsequent surgical interventions.

\section{Surgical procedure}

MIDCAB is a collective term for coronary artery bypass grafting $(\mathrm{CABG})$ procedures that are performed through small incisions as an alternative to median sternotomy. In RA-MIDCAB, harvesting of one or both IMA is performed using the da Vinci Xi robotic system (Intuitive Surgical Inc., Sunnyvale, CA, USA), followed by performance of the anastomosis under direct vision through a small $(3-4 \mathrm{~cm})$ left anterior thoracotomy at the fourth intercostal space. The entire procedure is performed on a beating heart and without the use of cardiopulmonary bypass. The detailed technique of RA-MIDCAB has been described elsewhere (8). Three ports were used to facilitate the robotic procedure while permitting the chest to be insufflated with $\mathrm{CO}_{2}$, including one camera port into the 4th intercostal space on the left anterior axillary line and two instrument ports in the 2 nd and 6th intercostal spaces close to the left midclavicular line. A soft tissue retractor and regular stabilization devices were used to enhance visualization through the thoracotomy. Quality of the anastomosis was assessed using intraoperative duplex and transit-time flow measurements with calculation of pulsatility index and diastolic and systolic flow. All operations were performed by 3 experienced cardiac surgeons.

\section{Statistical analysis}

The Shapiro-Wilk test was used to evaluate normality of data. Continuous variables are expressed as mean $\pm \mathrm{SD}$ or median (IQR), and differences between groups were tested using the nonparametric Kruskal-Wallis test or the parametric one-way ANOVA test according to data distribution. Pairwise comparisons were made and correction for multiple testing was applied using the Dunn test or Fisher least significant difference test, respectively. Categorical variables are expressed as numbers (\%), and differences were assessed with the Chi-squared test.

Logarithmic regression analysis was used to analyze learning curves based on surgical time. To evaluate the trends in the complications rates after RA-MIDCAB, we made use of the sequential probability CUSUM failure analysis. These were assessed for both MACCE and other complications. Expected and unacceptable rates were set at $12 \%$ and $20 \%$, respectively, for MACCE and at $40 \%$ and $60 \%$ for other complications, based on our prior experience and published data in CABG (9-13). These numbers have been defined to reflect the evolution in complication rates that a center might expect when transitioning from conventional CABG to RA-MIDCAB. Learning curves and CUSUM analyses were examined for all 300 procedures. Furthermore, a priori defined subgroup analyses were performed based on: tercile, type of construct, and hybrid vs. non-hybrid procedures. Because it was found that learning curves were restricted to the first 100 procedures, only the first 100 procedures for each of these subgroups were included in the subgroup analyses.

All tests were two-sided, and a $\mathrm{P}$ value less than 0.05 was deemed statistically significant. All analyses have been performed using SPSS software version 26 (SPSS Inc.) and R Statistical Software (version 4.0.2, Foundation for Statistical Computing, Vienna, Austria).

\section{Results}

Between July 1, 2015 and December 31, 2019, 300 RAMIDCAB surgeries and a total of 440 anastomoses were performed. Most surgeries comprised a single anastomosis $(\mathrm{n}=180,60.0 \%)$. In $111(37.0 \%)$ cases, 2 anastomoses were made and in $6(2.0 \%)$ cases 3 anastomoses. Of these 300 surgeries, $185(61.7 \%)$ consisted of a SIMA with 1 distal anastomosis, $86(28.7 \%)$ of a SIMA with 2 distal anastomoses, and $29(9.7 \%)$ consisted of BIMA or more than 2 distal anastomoses. In $70(23.3 \%)$ cases, RA$\mathrm{MIDCAB}$ was performed as part of a hybrid procedure.

\section{Demographics}

Demographic characteristics of the study population are described in Table 1 . The majority of the patients were male $(82.3 \%)$. The mean age was $66 \pm 10$ years, with a range of 33 to 90 years. Patients in the first tercile were significantly more often smokers (54\%) compared to the second 
Table 1 Demographic variables of the full study population

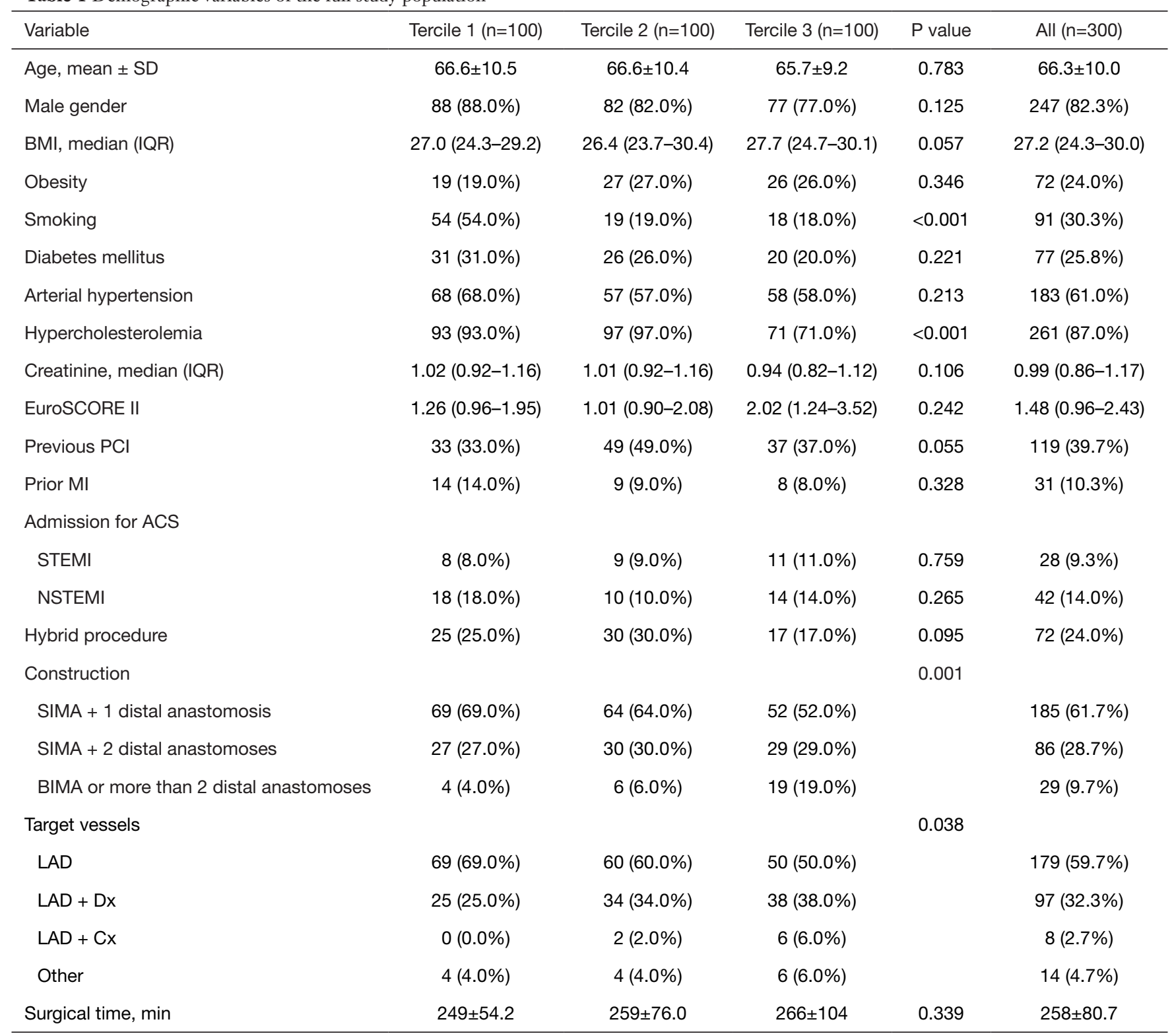

$\mathrm{P}<0.05$ is considered statistically significant. ACS, acute coronary syndrome; BIMA, bilateral internal mammary arteries; BMI, body mass index; IQR, interquartile range; MI, myocardial infarction; NSTEMI, non-ST-elevation myocardial infarction; PCI, percutaneous coronary intervention; SD, standard deviation; SIMA, single internal mammary artery; STEMI, ST-elevation myocardial infarction.

$(19.0 \%, \mathrm{P}<0.001)$ and third tercile $(18.0 \%, \mathrm{P}<0.001)$. The proportion of patients with hypercholesterolemia in the third tercile was significantly lower $(71.0 \%)$ compared to the first $(93.0 \%, \mathrm{P}<0.001)$ and second tercile $(97.0 \%$, $\mathrm{P}<0.001)$. Furthermore, the third tercile included more complex operations, as illustrated by a higher proportion of procedures involving BIMA or more than 2 distal anastomoses $(19.0 \%)$ compared to the first $(4.0 \%, \mathrm{P}<0.001)$ and second $(6.0 \%, \mathrm{P}=0.015)$ tercile. All other demographics were similar between the three terciles.

\section{Learning curves}

The mean surgical time for all operations was $258 \pm 81$ minutes, ranging from 127 to 821 minutes. The mean duration was $249 \pm 54$ minutes in the first tercile, $259 \pm 76$ minutes in the 
A

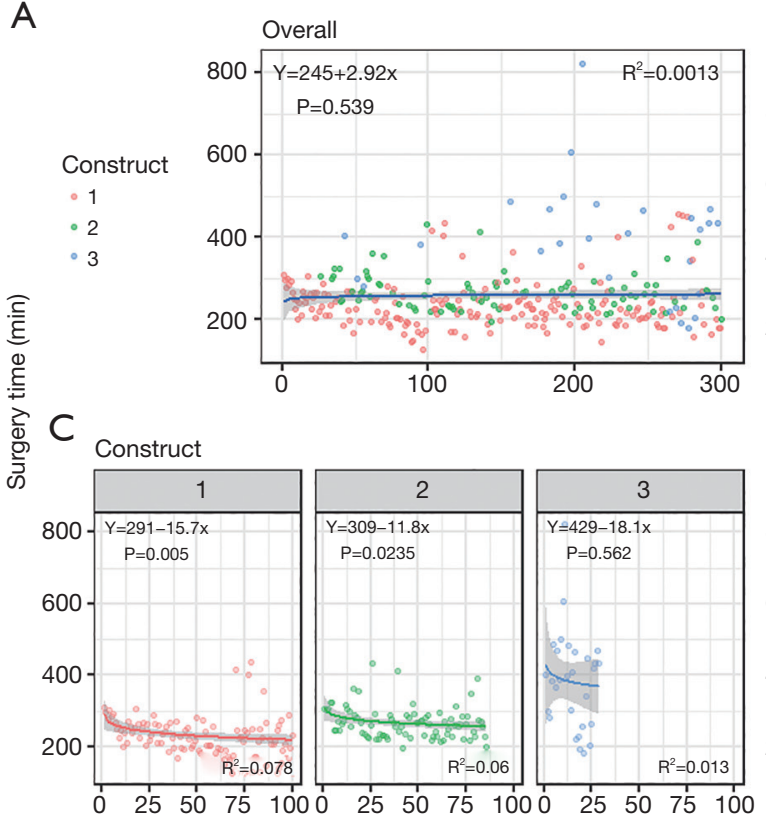

B Tercile

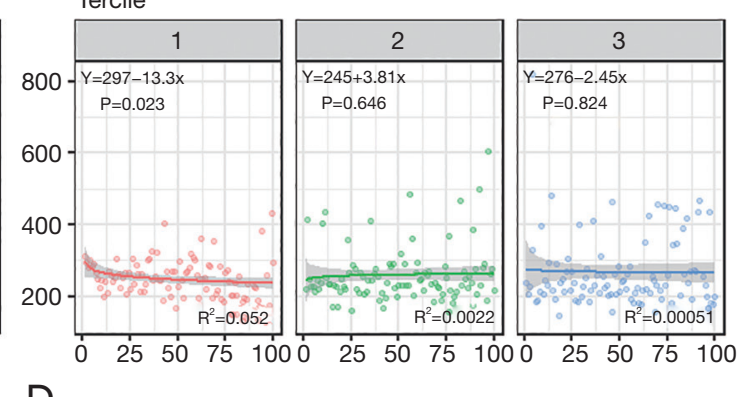

D Hybrid

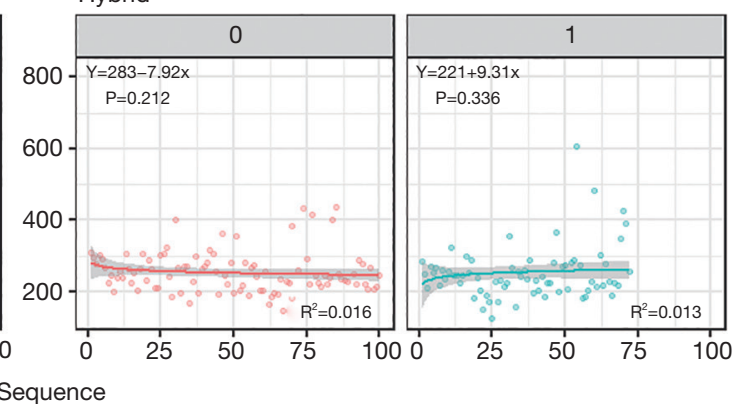

Figure 1 Learning curves. (A) For all 300 consecutive operations; (B,C,D) for the first 100 procedures of subgroups defined based on (B) tercile, (C) type of construct, (D) and hybrid versus non-hybrid procedures. Type of construct was subdivided into procedures including (I) SIMA +1 distal anastomosis, (II) SIMA +2 distal anastomoses, and (III) BIMA or more than 2 distal anastomoses. SIMA, single internal mammary artery; BIMA, bilateral internal mammary artery.

second, and $266 \pm 104$ minutes in the third. A learning curve was only observed in the first tercile $[y=297-13.3 \times \log (x)$, $\left.\mathrm{P}=0.023, \mathrm{R}^{2}=0.052\right]$ (Figure 1).

With regard to type of construct, procedures consisting of SIMA with 1 distal anastomosis had a mean surgical time of $234 \pm 58$ minutes, which showed a significant learning curve in the first 100 procedures of this type $\left[y=291-15.7 \times \log (x), P=0.005, R^{2}=0.072\right]$. Similarly, SIMA with 2 distal anastomoses had a mean surgical time of $268 \pm 45$, with a significant decrease over time $\left[y=309-11.8 \times \log (x), P=0.024, R^{2}=0.060\right]$. For the group with BIMA or more than 2 distal anastomoses, mean surgical time was $384 \pm 137$ minutes, but a significant learning curve could not be observed.

Mean surgical time was $260 \pm 81$ minutes for the hybrid group and $252 \pm 74$ minutes for the non-hybrid group. There was no significant learning curve in either of these groups.

\section{MACCE}

A total of 14 MACCEs were observed within 1 year after the operation (Table 2). One-year mortality occurred in
11 patients (3.7\%), with 6 deaths in the first, 3 in the second and 2 in the third tercile. Of these, 3 (1.0\%) occurred within 30 days after the operation ( 1 in the first and 2 in the third tercile). Two repeat revascularizations were required, of which 1 in the second and 1 in the third tercile. There was $1 \mathrm{MI}$ in the second tercile. No strokes occurred.

CUSUM analysis showed that the cumulative rate of MACCE never crossed the lines for unacceptable rates (Figure 2). Rather, after a short initial phase with the cumulative rate of MACCE close to the baseline, the lower $95 \%$ confidence boundary was crossed after 50 cases, indicating improvement in safety. Similar findings were observed in the subgroup analyses, except for hybrid procedures and procedures involving BIMA or more than 2 distal anastomoses, where the lower $95 \%$ confidence boundary was not crossed (Figure 2).

\section{Other complications}

Overall, 139 other complications were reported (Table 2). Of these, 40 occurred in the first tercile, 47 in the second tercile, and 52 in the third tercile. Prolonged ventilation 
Table 2 Summary of surgical complications in the full study population

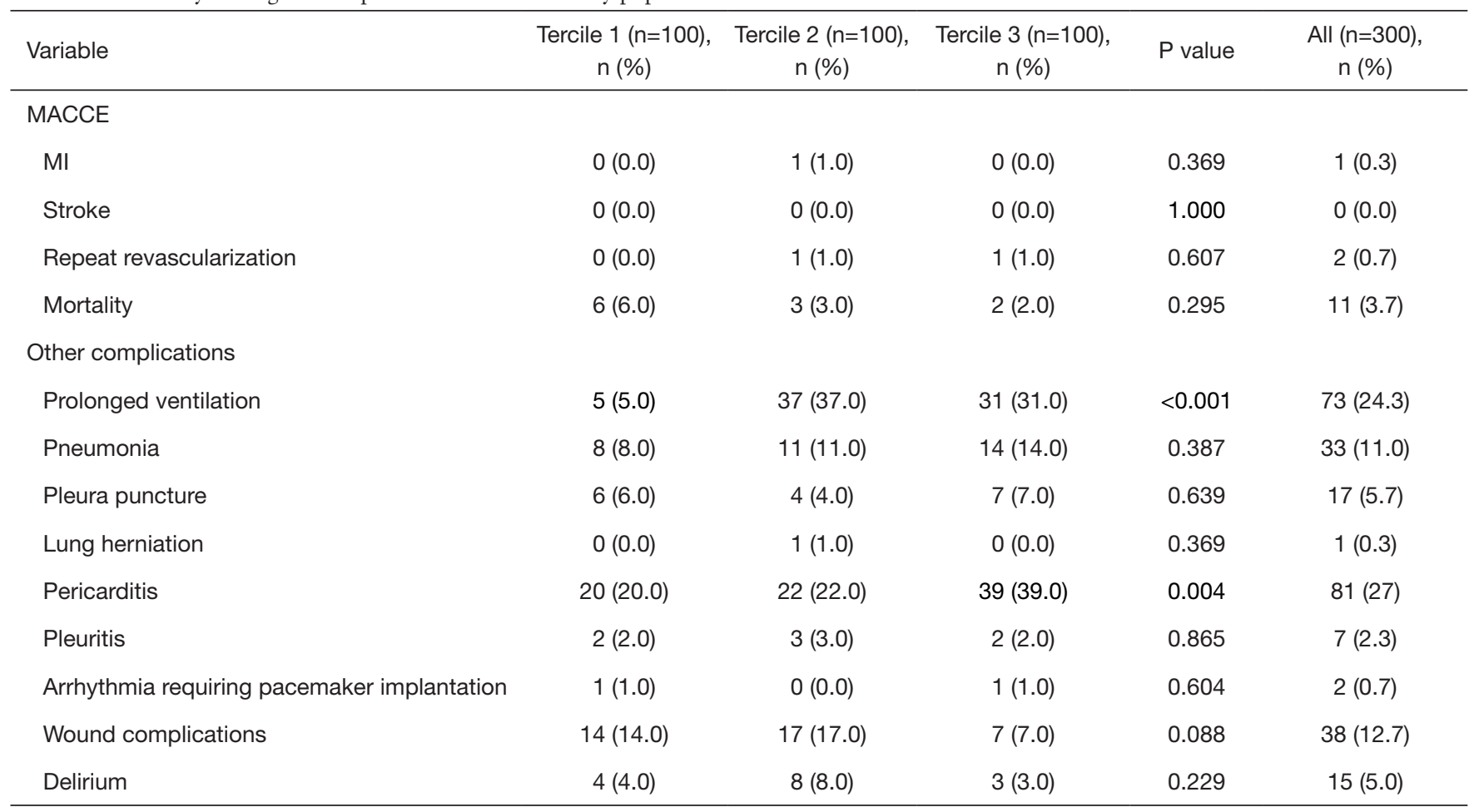

$\mathrm{P}<0.05$ is considered statistically significant. MACCE, major adverse cardiac and cerebrovascular events; MI, myocardial infarction.

was less frequent in the first tercile (5.0\%) compared to the second $(37.0 \%, \mathrm{P}<0.001)$ and third $(31.0 \%, \mathrm{P}<0.001)$. On the other hand, the occurrence of pericarditis significantly increased in the third tercile $(39.0 \%)$, compared to the first $(20.0 \%, \mathrm{P}=0.009)$ and second tercile $(22.0 \%, \mathrm{P}=0.027)$ in the third tercile. All other complications were evenly distributed across the three terciles.

CUSUM analysis revealed that the cumulative rate of minor events never crossed the lines for unacceptable rates (Figure 3). Rather, the lower $95 \%$ confidence boundary was crossed after 50 cases, indicating improvement in safety. However, subgroup analyses revealed that this safety advantage was limited to the first and second tercile, SIMA with 1 distal anastomosis, and non-hybrid procedures (Figure 3). The lower $95 \%$ confidence boundary was not crossed for procedures in the third tercile, for those involving SIMA with 2 distal anastomoses, BIMA or more than 2 distal anastomoses, and for hybrid procedures.

\section{Discussion}

Learning curves are inevitably encountered when first implementing an innovative and complex surgical technique.
Nevertheless, a cluster of failures or complications should be detected early, but not deter learning, to ensure safe implementation. In this study, we applied learning curves and CUSUM analysis to track the learning process in RAMIDCAB. A learning curve was only observed in the first tercile and was restricted mainly to procedures involving SIMA with 1 or 2 distal anastomoses. Furthermore, the lower $95 \%$ confidence boundary of the CUSUM curves for MACCE and other complications were crossed after 50 cases, indicating improvement in safety. These results suggest that integration of RA-MIDCAB in the surgical landscape can be safely achieved and complication rates can quickly be reduced below those expected in conventional CABG.

\section{RA-MIDCAB}

CABG is an established approach for the treatment of severe coronary artery disease but is associated with morbidity related to median sternotomy and cardiopulmonary bypass (14). In an attempt to improve short- and longterm outcomes after the procedure, minimally invasive techniques for myocardial revascularization have been 

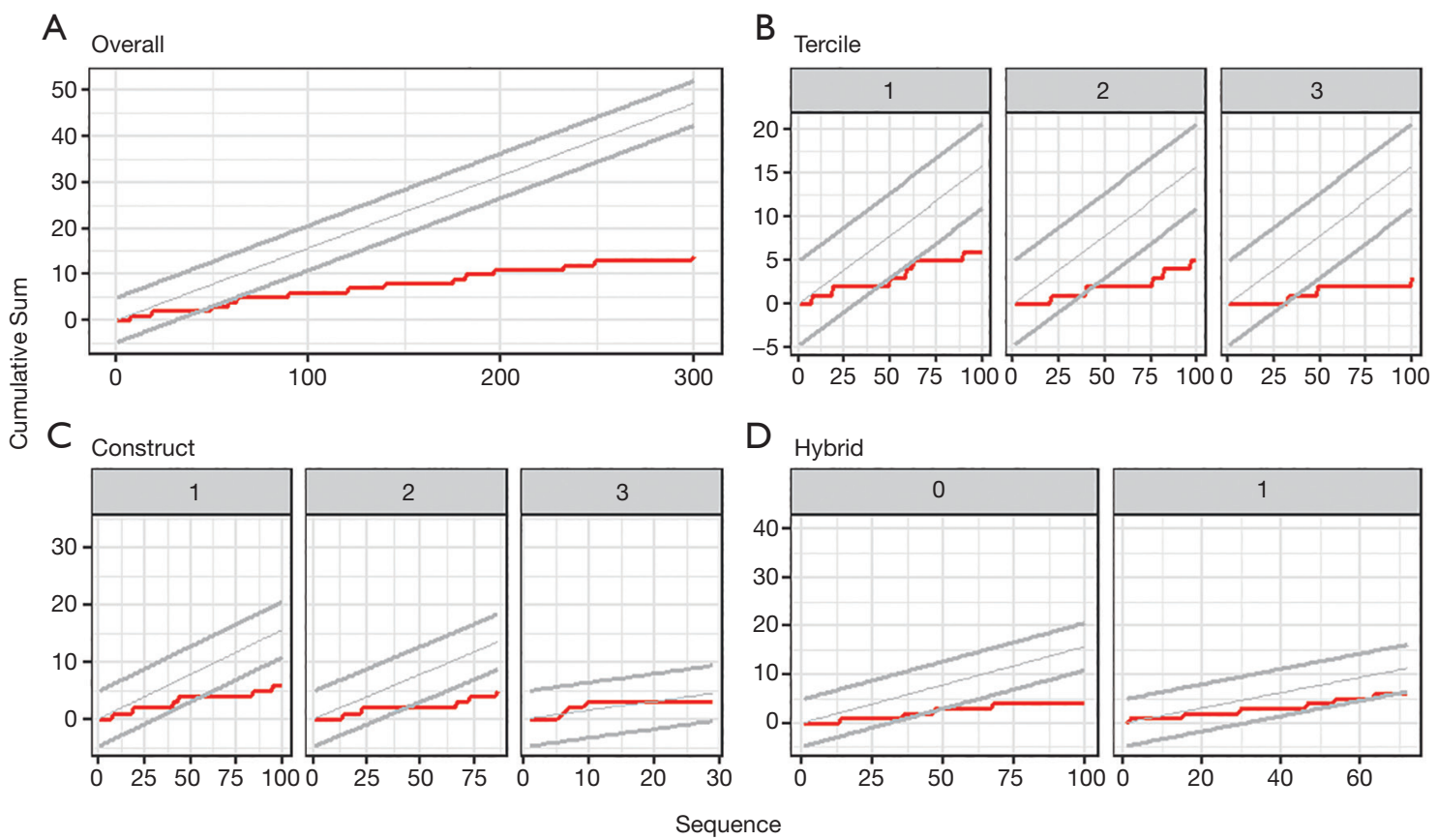

D Hybrid
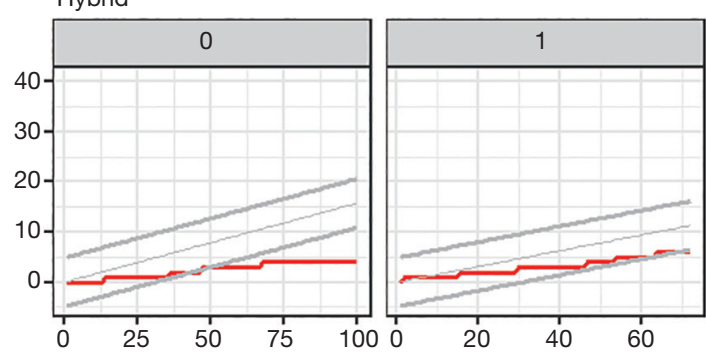

Figure 2 CUSUM analyses for MACCE complications. (A) For all 300 consecutive operations; (B,C,D) for the first 100 procedures of subgroups defined based on (B) tercile, (C) type of construct, (D) and hybrid versus non-hybrid procedures. Type of construct was subdivided into procedures including (I) SIMA +1 distal anastomosis, (II) SIMA +2 distal anastomoses, and (III) BIMA or more than 2 distal anastomoses. The red line represents the cumulative sum of complications, while the expected rate of complications and its $95 \%$ confidence boundaries are represented by the grey lines. CUSUM, cumulative sum; BIMA, bilateral internal mammary artery; MACCE, major adverse cardiac and cerebrovascular events; SIMA, single internal mammary artery.

introduced in the past two decades. During RA-MIDCAB, one or both internal mammary arteries are harvested using a robotic system and anastomoses are performed under direct vision through a small anterior thoracotomy. The entire procedure is performed on a beating heart, thus abandoning the detrimental effects of cardiopulmonary bypass on risk of embolization, inflammation, and acute renal failure (15).

Previous reports have demonstrated that RA-MIDCAB is a safe and efficacious alternative to traditional CABG, providing favorable short-term outcomes and longterm graft patency $(16,17)$. Additional advantages of the technique include reduced need for blood transfusion, shorter hospital stay, and improved quality of life (18). However, despite having been around for more than two decades, the technique only represents $1 \%$ of all CABG surgeries performed each year (15). One of the main barriers to wide-spread implementation is cost: depending on the calculation methods, RA-MIDCAB would generate an extra cost of $6-13 \%$ (19). However, it has been suggested that improvements in postoperative quality of life and a more expeditious return to work might make robotic surgery overall cost-effective (20). Another barrier is the issue of increased technical and logistical complexity, as well as limited supporting evidence for the procedure. Creating anastomoses on a beating heart and manipulation of the heart through a small thoracotomy are challenging and require advanced surgical skills (21). Furthermore, hemodynamic management and maintenance of single-lung ventilation during the procedure depend on considerable experience and training of anesthesiologists and nursing staff (22).

As a result, RA-MIDCAB provides a unique model to investigate learning processes. It creates a setting where all surgeons, anesthesiologists, and other surgical staff are confronted by the technical and logistic challenges of a new procedure, relying on each other's assistance and knowledge. In the present study, we found that that the learning curve of our center collectively showed a stabilization in the second tercile while a significant reduction of complications occurred after the $50^{\text {th }}$ procedure. These results seem to 

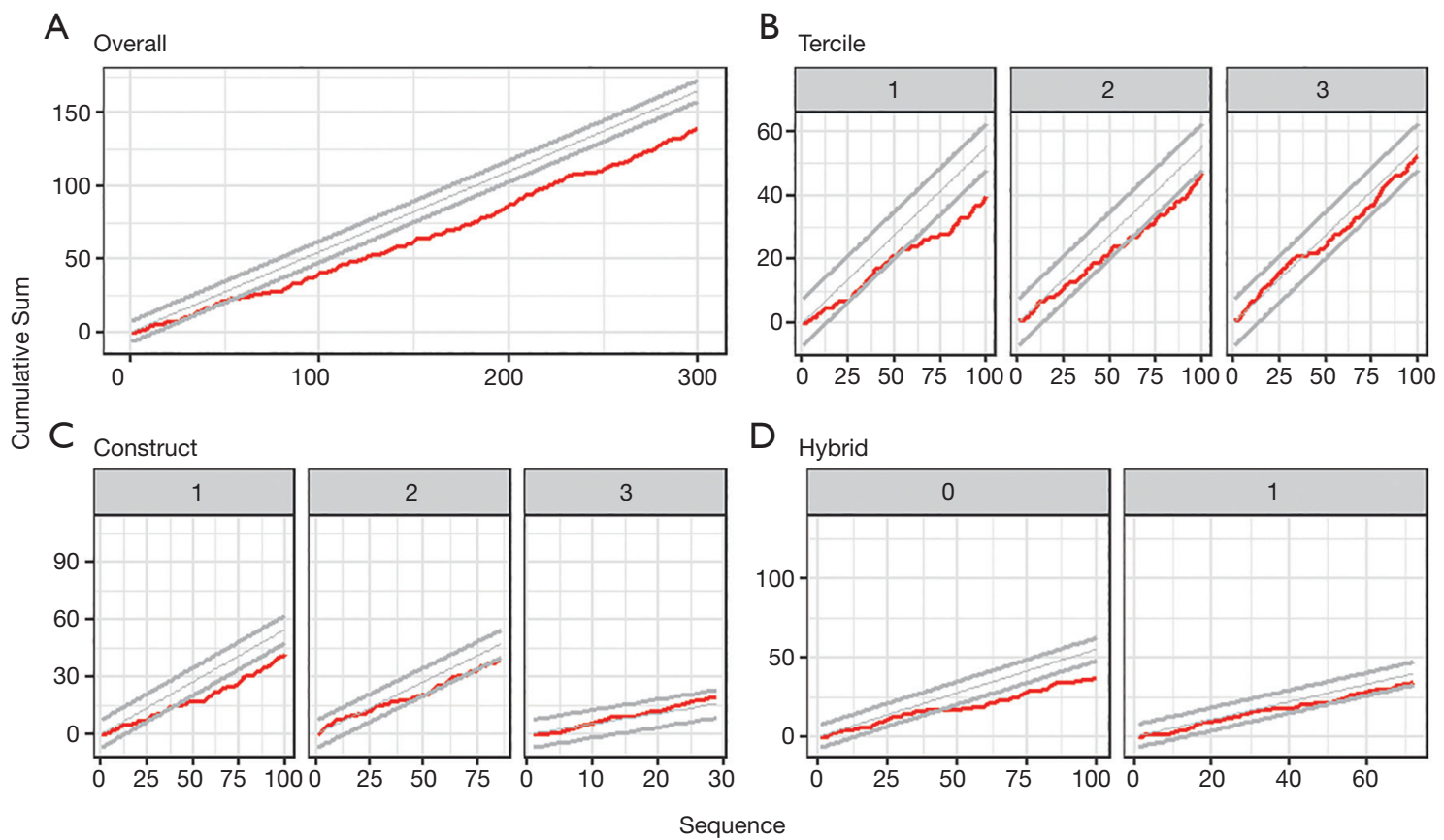

D Hybrid
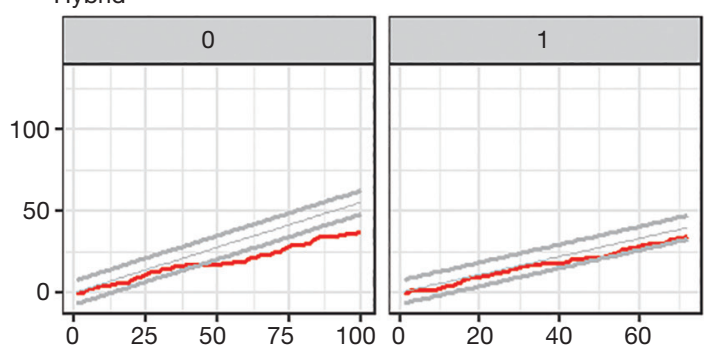

Figure 3 CUSUM analyses for other complications. (A) For all 300 consecutive operations; (B,C,D) for the first 100 procedures of subgroups defined based on (B) tercile, (C) type of construct, (D) and hybrid versus non-hybrid procedures. Type of construct was subdivided into procedures including (I) SIMA +1 distal anastomosis, (II) SIMA +2 distal anastomoses, and (III) BIMA or more than 2 distal anastomoses. The red line represents the cumulative sum of complications, while the expected rate of complications and its $95 \%$ confidence boundaries are represented by the grey lines. CUSUM, cumulative sum; BIMA, bilateral internal mammary artery; SIMA, single internal mammary artery.

suggest that about 50 operations are required to overcome the learning process and obtain favorable results with RAMIDCAB.

Of note, the surgical team at our institution had extensive prior experience with off-pump CABG (OPCAB). This implies that the surgeons had experience with anastomotic suturing on a beating heart, cardiac stabilizer devices, and apical suction devices. Furthermore, standardized protocols for hemodynamic management during off-pump procedures were already in place. In addition, the surgeons and anesthesiologists received advanced training in aspects of minimally invasive cardiac surgery before implementing the RA-MIDCAB program at our center. The fact that no increase in the complication rate during the beginning phase was seen, might at least in part be attributable to this prior experience.

\section{Learning curves}

Surgical times do not usually play a decisive role in patient outcomes, as long as they are not excessively long. Nonetheless, surgical times have been shown to reflect the cumulative experience of the full surgical team $(23,24)$. Thus, learning curves can act as a useful proxy for improvements in performance of a surgical team (25). Based on our study, most improvements in surgical time were observed within the first 100 procedures, as evidenced by the absence of a significant learning curve in later procedures.

Although no learning curve could be observed for procedures with BIMA or more than 2 distal anastomoses, it might be argued that this was due to the small number of cases in this group $(n=29)$. To test this hypothesis, we conducted a sensitivity analysis where a learning curve was constructed based on the first 29 procedures involving SIMA with 1 distal anastomosis. A significant learning curve was still observed $\left[\mathrm{y}=305-20 \times \log (\mathrm{x}), \mathrm{P}=0.003, \mathrm{R}^{2}=0.290\right]$, indicating that if a clear learning curve would have been present in procedures with BIMA or more than 2 distal anastomoses, this should have been observed within our dataset. However, the learning curve might have been masked either due to earlier experience with RA-MIDCAB 
or because it takes longer to significantly reduce surgical time in these more complex procedures.

\section{Complications}

CUSUM analysis is a powerful statistical technique that allows tracking of changes in peri-operative mortality and morbidity in the course of implementation of a new surgical technique (26). As it can be repeated as often as desired, even after each operation, it provides an almost real-time monitoring of surgical performance. Such information may be critically important to those centers that are planning to adopt an RA-MIDCAB program. From an educational perspective, CUSUM analysis may be helpful in establishing dedicated training programs and monitoring individual surgeon's complication rates.

Our observed number of operations to reach a turning point toward a lower complication rate was 50 procedures. It needs to be taken into account that the operations in our study were performed by a surgical team with extensive prior experience in OPCAB. Furthermore, the introduction of RA-MIDCAB was supported and supervised by world experts from other centers. One can conclude from our observations that, in experienced hands, the learning curve for RA-MIDCAB can be overcome relatively quickly.

One-year mortality $(3.7 \%)$ was comparable with other reports of RA-MIDCAB (1.4-4\%) (27-29), and 30-day mortality $(1.0 \%)$ tended to be lower than predicted by the EuroSCORE II (1.5\%). In addition, acute coronary syndrome was a common indication for RA-MIDCAB in our experience, and progressively patients with multivessel disease, history of transplantation, or those scheduled for redo surgery were included. A study by Lichtenberg et al. (30) found that a mortality of $3.6 \%$ can be expected for RAMIDCAB in patients with multivessel disease. Importantly, no periprocedural stroke was reported in any of the 300 patients in our study, which might result from the fact that RA-MIDCAB abandons the need for aortic crossclamping.

Other complications were mainly driven by pericarditis, prolonged ventilation, and wound complications. Pericarditis was more common in the third tercile, probably reflecting our change in practice in the past years to maintain a lower threshold of clinical suspicion of this complication when patients report chest pain or other nonspecific symptoms after the procedure. As such, more cases of pericarditis might have been diagnosed in the third tercile compared to the first and second. The higher incidence of prolonged ventilation in the last two terciles might be due to the introduction of more complex procedures in higherrisk patients. Furthermore, procedures including BIMA or more than 2 distal anastomoses were mainly introduced in the two last terciles (Figure 1A); in these patients, our policy was to send them to the intensive care unit for close monitoring out of precaution, thus explaining the higher proportion of prolonged ventilation. Similarly, the observation that the reduction in complications was less pronounced for hybrid procedures might be explained by the higher risk profile of these patients (31). Lastly, it should be highlighted that wound complications included issues ranging from superficial wound problems such as local redness and minimal drainage to deep wound complications and dehiscence at the thoracotomy or port sites (7).

\section{Perspectives on safe implementation of RA-MIDCAB}

As suggested above, optimal outcomes with implementation of RA-MIDCAB can be ensured by means of appropriate training and collective experience of the surgical team (32). Formal training courses to obtain specific skills required for RA-MIDCAB and assistance from expert practitioners during the first procedures are thus recommended. Furthermore, careful patient selection during the initial phase of the program, restricting to more simple cases, is essential. Finally, institutional factors such as prior experience of the team with OPCAB and familiarity of anesthesiologists with hemodynamic management during off-pump cardiac surgery should be considered. Keeping these conditions in mind, we believe that RA-MIDCAB can be implemented safely and efficiently.

Future research efforts should focus on improving surgical technique, developing novel technologies to assist RA-MIDCAB, establishing guidelines for the optimal implementation of RA-MIDCAB programs, and investigating tools such as CUSUM analysis which can help to monitor safety in real-time during implementation of new techniques and as part of the education of residents and fellows.

\section{Limitations}

A possible limitation of this study is the small sample size of the subanalyses. However, we specifically aimed to capture the introductory phase of RA-MIDCAB at our institution, as this gives an exclusive insight into how a center starts to accumulate experience. Furthermore, due to 
the retrospective nature of this study, data on complications were retrieved through electronic medical records. Therefore, there might have been heterogeneity in the reporting of certain outcomes. Lastly, the interval between the different procedures might have had an effect on the learning curve and complication rate, but this could not be accounted for.

\section{Conclusions}

In conclusion, these results suggest that integration of RAMIDCAB in the surgical landscape can be safely achieved and complication rates can quickly be reduced below those expected in traditional CABG. Collective experience plays a key role in overcoming the learning curve when more complex procedures and cases are introduced. Real-time monitoring of the learning process using learning curves and CUSUM analysis may facilitate this process.

\section{Acknowledgments}

J. Van den Eynde was supported by a Fellowship of the Belgian American Educational Foundation.

Funding: None.

\section{Footnote}

Reporting Checklist: The authors have completed the STROBE reporting checklist. Available at https://dx.doi. org/10.21037/jtd-21-775

Data Sharing Statement: Available at https://dx.doi. org/10.21037/jtd-21-775

Conflicts of Interest: All authors have completed the ICMJE uniform disclosure form (available at https://dx.doi. org/10.21037/jtd-21-775). WO is a proctor for Medtronic for multivessel CABG and MIDCAB. FPS participates in case observation and speakers bureau with Intuitive Surgical, Inc. The other authors have no conflicts of interest to declare.

Ethical Statement: The authors are accountable for all aspects of the work in ensuring that questions related to the accuracy or integrity of any part of the work are appropriately investigated and resolved. The study was conducted in accordance with the Declaration of Helsinki (as revised in 2013). The study was approved by local ethics board of the University Hospitals Leuven (No.: S64856) and informed consent was taken from all individual participants.

Open Access Statement: This is an Open Access article distributed in accordance with the Creative Commons Attribution-NonCommercial-NoDerivs 4.0 International License (CC BY-NC-ND 4.0), which permits the noncommercial replication and distribution of the article with the strict proviso that no changes or edits are made and the original work is properly cited (including links to both the formal publication through the relevant DOI and the license). See: https://creativecommons.org/licenses/by-nc-nd/4.0/.

\section{References}

1. Holzhey DM, Seeburger J, Misfeld M, et al. Learning minimally invasive mitral valve surgery: a cumulative sum sequential probability analysis of 3895 operations from a single high-volume center. Circulation 2013;128:483-91.

2. De Praetere H, Verbrugghe P, Rega F, et al. Starting minimally invasive valve surgery using endoclamp technology: safety and results of a starting surgeon. Interact Cardiovasc Thorac Surg 2015;20:351-8.

3. Murzi M, Cerillo AG, Bevilacqua S, et al. Traversing the learning curve in minimally invasive heart valve surgery: a cumulative analysis of an individual surgeon's experience with a right minithoracotomy approach for aortic valve replacement. Eur J Cardiothorac Surg 2012;41:1242-6.

4. Pettinari M, Navarra E, Noirhomme P, et al. The state of robotic cardiac surgery in Europe. Ann Cardiothorac Surg 2017;6:1-8.

5. Vandenbroucke JP, von Elm E, Altman DG, et al. Strengthening the Reporting of Observational Studies in Epidemiology (STROBE): explanation and elaboration. PLoS Med 2007;4:e297.

6. Thygesen K, Alpert JS, Jaffe AS, et al. Fourth Universal Definition of Myocardial Infarction (2018). Circulation 2018;138:e618-51.

7. Van den Eynde J, Heeren A, Szecel D, et al. Skeletonisation contributing to a reduction of sternal wound complications: a retrospective study in $\mathrm{OPCAB}$ patients. J Cardiothorac Surg 2019;14:162.

8. Bonatti J, Wallner S, Crailsheim I, et al. Minimally invasive and robotic coronary artery bypass grafting-a 25year review. J Thorac Dis 2021;13:1922-44.

9. Serruys PW, Morice MC, Kappetein AP, et al. Percutaneous coronary intervention versus coronary-artery 
bypass grafting for severe coronary artery disease. $\mathrm{N}$ Engl J Med 2009;360:961-72.

10. Magovern JA, Sakert T, Magovern GJ, et al. A model that predicts morbidity and mortality after coronary artery bypass graft surgery. J Am Coll Cardiol 1996;28:1147-53.

11. Cao C, Manganas C, Bannon P, et al. Drug-eluting stents versus coronary artery bypass graft surgery in left main coronary artery disease: a meta-analysis of early outcomes from randomized and nonrandomized studies. J Thorac Cardiovasc Surg 2013;145:738-47.

12. Wang XW, Qu C, Huang C, et al. Minimally invasive direct coronary bypass compared with percutaneous coronary intervention for left anterior descending artery disease: a meta-analysis. J Cardiothorac Surg 2016;11:125.

13. Crawford TC, Magruder JT, Grimm JC, et al. Complications After Cardiac Operations: All Are Not Created Equal. Ann Thorac Surg 2017;103:32-40.

14. Van den Eynde J, Bennett J, McCutcheon K, et al. Heart team 2.0: A decision tree for minimally invasive and hybrid myocardial revascularization. Trends Cardiovasc Med 2020. [Epub ahead of print]. doi: 10.1016/j.tcm.2020.07.005.

15. Gaudino M, Bakaeen F, Davierwala P, et al. New Strategies for Surgical Myocardial Revascularization. Circulation 2018;138:2160-8.

16. Doulamis IP, Spartalis E, Machairas N, et al. The role of robotics in cardiac surgery: a systematic review. J Robot Surg 2019;13:41-52.

17. Kitahara H, Nisivaco S, Balkhy HH. Graft Patency after Robotically Assisted Coronary Artery Bypass Surgery. Innovations (Phila) 2019;14:117-23.

18. Raja SG, Garg S, Rochon M, et al. Short-term clinical outcomes and long-term survival of minimally invasive direct coronary artery bypass grafting. Ann Cardiothorac Surg 2018;7:621-7.

19. Barbash GI, Glied SA. New technology and health care costs--the case of robot-assisted surgery. N Engl J Med 2010;363:701-4.

20. Morgan JA, Thornton BA, Peacock JC, et al. Does robotic technology make minimally invasive cardiac surgery too expensive? A hospital cost analysis of robotic and conventional techniques. J Card Surg 2005;20:246-51.

21. Yasuda S, Van den Eynde J, Vandendriessche K, et al. Implementation of a beating heart system for training in off-pump and minimally invasive coronary artery bypass. BMC Surg 2021;21:26.

22. Song HK, Petersen RJ, Sharoni E, et al. Safe evolution towards routine off-pump coronary artery bypass: negotiating the learning curve. Eur J Cardiothorac Surg
2003;24:947-52.

23. Maruthappu M, Duclos A, Lipsitz SR, et al. Surgical learning curves and operative efficiency: a cross-specialty observational study. BMJ Open 2015;5:e006679.

24. Elbardissi AW, Duclos A, Rawn JD, et al. Cumulative team experience matters more than individual surgeon experience in cardiac surgery. J Thorac Cardiovasc Surg 2013;145:328-33.

25. Burt BM, ElBardissi AW, Huckman RS, et al. Influence of experience and the surgical learning curve on long-term patient outcomes in cardiac surgery. J Thorac Cardiovasc Surg 2015;150:1061-7, 1068.e1-3.

26. Novick RJ, Fox SA, Stitt LW, et al. Assessing the learning curve in off-pump coronary artery surgery via CUSUM failure analysis. Ann Thorac Surg 2002;73:S358-62.

27. Birla R, Patel P, Aresu G, et al. Minimally invasive direct coronary artery bypass versus off-pump coronary surgery through sternotomy. Ann R Coll Surg Engl 2013;95:481-5.

28. Holzhey DM, Jacobs S, Mochalski M, et al. Seven-year follow-up after minimally invasive direct coronary artery bypass: experience with more than 1300 patients. Ann Thorac Surg 2007;83:108-14.

29. Mehran R, Dangas G, Stamou SC, et al. One-year clinical outcome after minimally invasive direct coronary artery bypass. Circulation 2000;102:2799-802.

30. Lichtenberg A, Klima U, Paeschke H, et al. Impact of multivessel coronary artery disease on outcome after isolated minimally invasive bypass grafting of the left anterior descending artery. Ann Thorac Surg 2004;78:487-91.

31. Harskamp RE, Brennan JM, Xian Y, et al. Practice patterns and clinical outcomes after hybrid coronary revascularization in the United States: an analysis from the society of thoracic surgeons adult cardiac database. Circulation 2014;130:872-9.

32. Van den Eynde J, Melly L, Torregrossa G, et al. Robotic Cardiac Surgery: What the Young Surgeon Should Know. Braz J Cardiovasc Surg 2020;35:VI-VIII.

Cite this article as: Van den Eynde J, Vaesen Bentein H, Decaluwé T, De Praetere H, Wertan MC, Sutter FP, Balkhy $\mathrm{HH}$, Oosterlinck W. Safe implementation of robotic-assisted minimally invasive direct coronary artery bypass: application of learning curves and cumulative sum analysis. J Thorac Dis 2021;13(7):4260-4270. doi: 10.21037/jtd-21-775 